\title{
THE THREE-SEPARATED-ARC PROPERTY OF FUNCTIONS IN A DISK
}

\author{
F. Bagemihl
}

Let $D$ be the open unit disk and $\Gamma$ be the unit circle in the complex plane, and suppose that $f(z)$ is a single-valued function in $D$ with values on the Riemann sphere. If $\zeta \in \Gamma$ and if $\Lambda$ is an arc at $\zeta$, then $C_{\Lambda}(f, \zeta)$ denotes the cluster set of $f$ at $\zeta$ along $\Lambda$. If there exist three $\operatorname{arcs} \Lambda_{1}, \Lambda_{2}, \Lambda_{3}$ at $\zeta$ such that

$$
\mathbf{C}_{\Lambda_{1}}(f, \zeta) \cap \mathbf{C}_{\Lambda_{2}}(f, \zeta) \cap \mathbf{C}_{\Lambda_{3}}(f, \zeta)=\emptyset,
$$

then $f$ is said to have the three-arc property at $\zeta$. If the three arcs can be taken to be mutually exclusive, we say that $f$ has the three-separated-arc property at $\zeta$; if they can be taken to be chords at $\zeta, f$ is said to have the three-chord property at $\zeta$.

There exists [3] a normal holomorphic function in $D$ that has the threeseparated-arc property at every point of $\Gamma$. It follows from [1, Theorem 4], however, that the set of points of $\Gamma$ at each of which a normal meromorphic function in $D$, and hence, in particular, a bounded holomorphic function in $D$, has the three-chord property, is of measure zero and first category. There does exist [6, Theorem 2] a meromorphic function in $D$ that has the three-chord property at each point of a perfect subset of $\Gamma$. The set of points on $\Gamma$ at each of which a meromorphic function of bounded characteristic in $D$ has the three-arc property is of measure zero [2, Theorem 2]; this holds then, in particular, for any function that is holomorphic and bounded in $D$. A consequence of [1, Theorem 3$]$ is that there is a bounded holomorphic function in $D$ having the three-chord property at each point of an enumerable subset of $\Gamma$.

There is [5, Theorem 4] a bounded holomorphic function in $D$, in the form of a Blaschke product, that has the three-arc property at each point of a perfect subset of $\Gamma$. It has been shown recently [4] that if a meromorphic function in $D$ has the three-arc property at a point of $\Gamma$, then it has the three-separated-arc property at that point. Hence

There exists a Blaschke product that has the three-separated-arc property at each point of a perfect subset of $\Gamma$. 
C.L. Belna has asked (in written communications):

Does there exist a bounded holomorphic function in $D$ having the three-chord property at each point of a nonenumerable subset of $\Gamma$ ?

If a function is continuous in $D$ and has the three-arc property at a point $\zeta \in \Gamma$, does it have the three-separated-arc property at $\zeta$ ?

We prove:

There exists a three-valued function in $D$ that has the three-arc property at a point $\zeta \in \Gamma$ but does not have the three-separated-arc property at $\zeta$.

It is easier to describe the construction of the function in a half-plane instead of a disk, so we define our function in the lower half of the complex plane, and take the point $\zeta$ to be the origin.

Let $\Lambda_{1}$ be the segment extending from $-1-i$ to $\zeta$, and $\Lambda_{2}$ be the segment extending from $1-i$ to $\zeta$. Denote by $\left(a_{n}\right)$ and $\left(b_{n}\right)$ sequences of points on $\Lambda_{1}$, $\Lambda_{2}$, respectively, with imaginary parts $-1 / n(n=1,2,3, \ldots)$. Define $\left(c_{n}\right)$ to be the sequence of points on $\Lambda_{2}$ with imaginary parts $\frac{1}{2}(-(1 / 2 n)-1 /(2 n+1))$ $(n=1,2,3 \ldots)$. Take the point $d$ to be $-i$. We let $\Lambda_{3}$ be the arc at $\zeta$ consisting of the segments

$$
d a_{2}, a_{2} c_{1}, c_{1} a_{4}, a_{4} c_{2}, c_{2} a_{6}, \ldots, a_{2 n} c_{n}, c_{n} a_{2 n+2}, \ldots
$$

Define the function $f(z)$, for $z$ in the lower half-plane, to have one of the three values $1,2,3$ at $z$, in the following way.

For $z$ on each of the open segments $a_{2 n-1} a_{2 n}(n=1,2,3, \ldots), f(z)=1$. For $z=a_{1}$ and for $z$ on each of the closed segments $a_{2 n} a_{2 n+1}(n=1,2,3, \ldots)$, $f(z)=2$. Then $f(z)$ is defined on $\Lambda_{1}$, and $C_{\Lambda_{1}}(f, \zeta)=\{1,2\}$.

For $z$ on each of the closed segments $b_{2 n-1} b_{2 n}(n=1,2,3, \ldots), f(z)=1$. For $z$ on each of the open segments $b_{2 n} b_{2 n+1}(n=1,2,3, \ldots), f(z)=3$. Then $f(z)$ is defined on $\Lambda_{2}$, and $C_{\Lambda_{2}}(f, \zeta)=\{1,3\}$.

For $z=d$, for $z$ on each of the open segments $a_{2 n} c_{n}(n=1,2,3, \ldots)$, for $z$ on each of the open segments $c_{n} a_{2 n+2}(n=1,2,3, \ldots)$, and for $z$ on the open segment $d a_{2}, f(z)=3$. Then $f(z)$ is defined on $\Lambda_{3}$, and (note that $f\left(a_{2 n}\right)=2$, $(n=1,2,3, \ldots)) C_{\Lambda_{3}}(f, \zeta)=\{2,3\}$.

For $z$ on the open segment $a_{1} d, f(z)=1$; for $z$ on the open segment $d b_{1}$, $f(z)=3$. For $z$ on each of the open segments $a_{2 n} b_{2 n}(n=1,2,3, \ldots), f(z)=1$.

For $z$ inside the triangle with vertices $a_{1}, a_{2}, d$, for $z$ inside each of the triangles with vertices $a_{2 n}, b_{2 n}, c_{n}(n=1,2,3, \ldots)$, and for $z$ inside each of the triangles with vertices $c_{n}, a_{2 n+1}, a_{2 n+2}(n=1,2,3, \ldots), f(z)=1$.

For $z$ inside each of the triangles with vertices $c_{n}, a_{2 n}, a_{2 n+1}(n=$ $1,2,3, \ldots), f(z)=2$.

For every $z$ inside the triangle $\Delta$ with vertices $a_{1}, b_{1}, \zeta$ at which $f$ has not yet been defined, $f(z)=3$. 
Suppose that $z=x+i y$ is a point in the lower half-plane in the exterior of $\Delta$. If $y \leq-1, f(z)=1$. Express the interval $(-1,0)$ as the union of three disjoint sets $A, B, C$, each everywhere dense in the interval. If $y \in A, f(z)=1$; if $y \in B, f(z)=2$; if $y \in C, f(z)=3$.

This completes the definition of $f(z)$ in the lower half-plane.

Since

$$
C_{\Lambda_{1}}(f, \zeta) \cap C_{\Lambda_{2}}(f, \zeta) \cap C_{\Lambda_{3}}(f, \zeta)=\{1,2\} \cap\{1,3\} \cap\{2,3\}=\emptyset,
$$

$f$ has the three-arc property at $\zeta$.

To show that $f$ does not have the three-separated-arc property at $\zeta$, suppose, to the contrary, that there exist three disjoint $\operatorname{arcs} \Sigma_{1}, \Sigma_{2}, \Sigma_{3}$ at $\zeta$ such that

$$
C_{\Sigma_{1}}(f, \zeta) \cap C_{\Sigma_{2}}(f, \zeta) \cap C_{\Sigma_{3}}(f, \zeta)=\emptyset .
$$

We observe the following:

1. From the way in which $f$ was defined, it is evident that $f$ does not have an asymptotic value at $\zeta$.

2. In order for (1) to hold, none of the three cluster sets in (1) can contain all three values $1,2,3$.

3. Hence, each of the three cluster sets in (1) consists of two values, and for (1) to hold, the three cluster sets must be the sets $\{1,2\},\{1,3\},\{2,3\}$.

4. If an arc at $\zeta$ has, in every neighborhood of $\zeta$, a subarc lying to the left of $\Lambda_{1}$ or a subarc lying to the right of $\Lambda_{2}$, then the cluster set of $f$ at $\zeta$ along that arc is $\{1,2,3\}$. Consequently, the arcs $\Sigma_{1}, \Sigma_{2}, \Sigma_{3}$ must eventually lie in the closure of $\Delta$.

5. Suppose that $C_{\Sigma_{1}}(f, \zeta)=\{1,2\}$. Then $\Sigma_{1}$ must contain all but a finite number of the points $a_{2 n}(n=1,2,3, \ldots)$.

6. Suppose that $C_{\Sigma_{2}}(f, \zeta)=\{2,3\}$. Then $\Sigma_{2}$ must also contain all but a finite number of the points $a_{2 n}(n=1,2,3, \ldots)$.

7. It follows that $\Sigma_{1} \cap \Sigma_{2} \neq \emptyset$, contradicting the supposition that $\Sigma_{1}$ and $\Sigma_{2}$ are disjoint.

Thus our supposition is untenable, and our result is proved. 


\section{References}

[1] BAgEminL, F.: Some results and problems concerning chordal principal cluster sets. Nagoya Math. J. 29, 1967, 7-18.

[2] BAgeminL, F.: The three-arc and three-separated-arc properties of meromorphic functions. - Nagoya Math. J. 53, 1974, 137-140.

[3] BageminL, F.: The three-separated-arc property of the modular function. - Nagoya Math. J. 61, 1976, 203-204.

[4] BageminL, F., and S. Nicol: The three-arc implies the three-separated-arc property. To appear.

[5] Bagemihl, F., G. Piranian, and G.S. Young: Intersections of cluster sets. - Bul. Inst. Politehn. Iaşi Sect. I 5, 1959, 29-34.

[6] Gresser, J.T.: On uniform approximation by rational functions with an application to chordal cluster sets. - Nagoya Math. J. 34, 1969, 143-148.

The University of Wisconsin-Milwaukee

Department of Mathematical Sciences

P.O. Box 413

Milwaukee, WI 53201

U.S.A.

Received 11 July 1990 